\title{
Noncommutative Supersymmetric Field Theories
}

\author{
Victor O. Rivelles \\ Instituto de Física, Universidade de São Paulo \\ Caixa Postal 66318, 05315-970, São Paulo - SP, Brazil \\ E-mail: rivelles@fma.if.usp.br
}

Received on 19 February, 2001

\begin{abstract}
We discuss some properties of noncommutative supersymmetric field theories which do not involve
\end{abstract} gauge fields. We concentrate on the renormalizability issue of these theories.

\section{Introduction}

Although string theory is quite well understood in the perturbative regime its formulation in a background independent way is almost unknown. There are many reasons for that. String theory has too many degrees of freedom. It is quite difficult to handle all of them together. It also includes the gravitational field which may have quantum fluctuations. And there are many sources for nonlocality which is also troublesome in any theory. One way out of these difficulties is to consider limits of string theory which have some of the troubles raised above but not all of them. This may allow us to understand better some aspects of string theory without the complications of the full theory.

One such a limit is the zero slope limit of the D3brane in the presence of a constant NS-NS field [1]. The low energy effective theory is a quantum field theory deformed in terms of the Moyal product over space-time. In noncommutative field theories the usual product of fields is replaced by the Moyal product of fields giving rise to nonlocal field theories [2]. Usually nonlocal field theories turn out to be not well defined but the nonlocality induced by the Moyal product is still tractable. It was found that the main characteristic of noncommutative field theories is the mixing of ultraviolet (UV) and infrared (IR) divergences due to its nonlocal structure [3]. As a consequence it is not clear that the properties of the usual commutative field theories are kept, without modifications, in their noncommutative counterparts. This gave rise to an intensive research of noncommutative field theories in Euclidean or Minkowski space-time.

One of the manifestations of the UV/IR mixing in the $\lambda \phi^{4}$ theory is as an infrared quadratic singularity in the propagator at one loop [3]. Although renormalizable up to two loops [4] it becomes non-renormalizable at higher loop orders. Models involving a complex scalar field may be non-renormalizable even at one loop [5]. So, noncommutativity seems to destroy the main characteristic of commutative field theories, i.e., their renormalizability.

In what follows we will discuss the inclusion of supersymmetry in such models and how it restores the renormalizability. We will concentrate on the WessZumino model in $3+1$ dimensions [6] and the supersymmetric non-linear sigma model in $2+1$ dimensions [7]. In this last case we will see that the noncommutativity also destroys the mechanism for dynamical mass generation of the fermionic sector, and we will show how supersymmetry helps to fix it.

\section{Noncommutative Spaces}

In quantum mechanics we have the usual commutation relations

$$
\begin{aligned}
& {\left[\hat{q}^{i}, \hat{p}^{j}\right]=i \hbar g^{i j}} \\
& {\left[\hat{q}^{i}, \hat{q}^{j}\right]=\left[\hat{p}^{i}, \hat{p}^{j}\right]=0 .}
\end{aligned}
$$

It is natural to consider noncommutative coordinates with commutation relations

$$
\left[\hat{q}^{i}, \hat{q}^{j}\right]=i \theta^{i j}
$$

where $\theta_{i j}$ is a constant of dimension $L^{2}$ which defines a noncommutativity scale. This breaks rotational (or Lorentz) symmetry but in the limit $\theta \rightarrow 0$ the symmetry is recovered. This is an example of a noncommutative space. It can be extended to space-time but we will consider noncommutativity only in the spatial coordinates since otherwise there are problems with unitarity [8].

We can understand heuristically how the UV and IR physics gets mixed. From Eq.(1) it follows that $\Delta \hat{q}^{i} \Delta \hat{p}^{j} \sim i g^{i j}$. In a similar way, from Eq.(3) it follows that $\Delta \hat{q}^{i} \Delta \hat{q}^{j} \sim i \theta^{i j}$ so we expect that $\Delta \hat{q} \sim \theta \Delta \hat{p}$. This means that high energy modes have drastic effects at large distances (or small energy processes). As we shall see, in quantum field theory this mixing manifests 
itself already at one loop level in the propagator of the fields.

Fields defined on such spaces are operator valued objects. It turns out to be more convenient to use fields which are not operator valued objects but just functions. This can be achieved through the use of the Weyl-Moyal correspondence [2]

$$
\hat{\Phi}(\hat{q}) \rightarrow \phi(x) \text {. }
$$

We associate to the operator valued field $\hat{\Phi}(\hat{q})$ a classical function $\phi(x)$ through its Fourier transform $\tilde{\phi}(p)$ as

$$
\hat{\Phi}(\hat{q})=\int d p e^{i p \hat{q}} \tilde{\phi}(p)
$$

The operator valued field $\hat{\Phi}$ satisfies

$$
\hat{\Phi}_{1}(\hat{q}) \hat{\Phi}_{2}(\hat{q})=\int d p_{1} d p_{2} e^{i\left(p_{1}+p_{2}\right) \hat{q}-\frac{1}{2} p_{1}^{\mu} p_{2}^{\nu} \theta_{\mu \nu}} \phi_{1}\left(p_{1}\right) \phi_{2}\left(p_{2}\right)
$$

hence

$$
\hat{\Phi}_{1}(\hat{q}) \hat{\Phi}_{2}(\hat{q}) \leftrightarrow\left(\phi_{1} \star \phi_{2}\right)(x),
$$

where

$$
\left(\phi_{1} \star \phi_{2}\right)(x) \equiv\left[e^{i \frac{1}{2} \theta^{\mu \nu} \frac{\partial}{\partial x^{\mu}} \frac{\partial}{\partial y^{\nu}}} \phi_{1}(x) \phi_{2}(y)\right]_{y=x},
$$

is the Moyal (or star) product. Then we can work on a commutative space in which the usual product of field is replaced by the Moyal product. Notice that the derivatives in the definition Eq.(8) makes the Moyal product non-local. Also, the Moyal commutator of the commutative coordinates $x^{\mu}$ gives

$$
\left[x^{\mu}, x^{\nu}\right]_{M B}=x^{\mu} \star x^{\nu}-x^{\nu} \star x^{\mu}=i \theta^{\mu \nu} .
$$

It can be easily verified the following properties of the Moyal product:

$$
\text { a) } e^{i k x} \star e^{i q y}=e^{i(k+q) x} e^{-i k \wedge q} \text {, }
$$

where $k \wedge q=\frac{1}{2} k^{\mu} \theta_{\mu \nu} q^{\nu}$.

$$
\text { b) } \quad(f \star g)(x)=\int d k d q \tilde{f}(k) \tilde{g}(q) e^{-i k \wedge q} e^{i(k+q) x} \text {, }
$$

where $\tilde{f}$ and $\tilde{g}$ are the Fourier components of $f$ and $g$, respectively.

$$
\begin{gathered}
c) \quad[(f \star g) \star h](x)=[f \star(g \star h)](x) . \\
\text { d) } \int d x(f \star g)(x)=\int d x(g \star f)(x)=\int d x f(x) g(x) . \\
\text { e) } \quad \int d x\left(f_{1} \star f_{2} \star \ldots f_{n}\right)(x)=\int d x\left(f_{n} \star f_{1} \star \ldots f_{n-1}\right)(x) . \\
f)(f \star g)^{*}=g^{*} \star f^{*} .
\end{gathered}
$$

\section{Noncommutative Scalar Field Theory}

Let us consider the massive scalar field in $D=3+1$ dimensions

[3], whose action is

$$
S=\int d^{4} x\left(\frac{1}{2} \partial_{\mu} \phi \star \partial^{\mu} \phi-\frac{m^{2}}{2} \phi \star \phi-\frac{g^{2}}{4 !} \phi \star \phi \star \phi \star \phi\right) .
$$

Using property d) it is seen that the propagator is not affected by the Moyal product. This is a generic property of noncommutative field theories. The vertex, however, must be symmetrized . In momentum space we have

$$
\begin{aligned}
& -\frac{g^{2}}{2} \int d^{4} x \phi \star \phi \star \phi \star \phi=-\frac{g^{2}}{6} \int d k_{1} d k_{2} d k_{3} d k_{4} \delta\left(k_{1}+k_{2}+k_{3}+k_{4}\right) \times \\
& {\left[\cos \left(\frac{1}{2} k_{1} \wedge k_{2}\right) \cos \left(\frac{1}{2} k_{3} \wedge k_{4}\right)+\cos \left(\frac{1}{2} k_{1} \wedge k_{3}\right) \cos \left(\frac{1}{2} k_{2} \wedge k_{4}\right)+\right.} \\
& \left.\cos \left(\frac{1}{2} k_{1} \wedge k_{4}\right) \cos \left(\frac{1}{2} k_{2} \wedge k_{3}\right)\right] \phi\left(k_{1}\right) \phi\left(k_{2}\right) \phi\left(k_{3}\right) \phi\left(k_{4}\right) .
\end{aligned}
$$


Then, the one loop correction for the two-point function is

$$
\frac{g^{2}}{3(2 \pi)^{4}} \int d^{4} k\left(1+\frac{1}{2} \cos (k \wedge p)\right) \frac{1}{k^{2}+m^{2}} .
$$

The first term is the usual one loop mass correction of the commutative theory (up to a factor $1 / 2$ ) which is quadratically divergent. The second term is not divergent due to the oscillatory nature of $\cos (k \wedge p)$. This shows that the nonlocality introduced by the Moyal product is not bad and leaves us with the same divergence structure of the commutative theory. To take into account the effect of the second term we regularize the integral using the Schwinger parametrization

$$
\frac{1}{k^{2}+m^{2}}=\int_{0}^{\infty} d \alpha e^{-\alpha\left(k^{2}+m^{2}\right)} e^{-\frac{1}{\Lambda^{2} \alpha}}
$$

where a cutoff $\Lambda$ was introduced. We find

$$
\Gamma^{(2)}=\frac{g^{2}}{48 \pi^{2}}\left[\left(\Lambda^{2}-m^{2} \ln \left(\frac{\Lambda^{2}}{m^{2}}\right)+\ldots\right)+\frac{1}{2}\left(\Lambda_{e f f}^{2}-m^{2} \ln \left(\frac{\Lambda_{e f f}^{2}}{m^{2}}\right)+\ldots\right)\right],
$$

where

$$
\Lambda_{e f f}^{2}=\frac{1}{\frac{1}{\Lambda^{2}}+\tilde{p}^{2}}, \quad \tilde{p}^{\mu}=\theta^{\mu \nu} p_{\nu}
$$

Note that when the cutoff is removed, $\Lambda \rightarrow \infty$, the noncommutative contribution remains finite providing a natural regularization. Also $\Lambda_{e f f}^{2}=\frac{1}{\tilde{p}^{2}}$ which diverges either when $\theta \rightarrow 0$ or when $\tilde{p} \rightarrow 0$.

The one loop effective action is then

$$
\int d^{4} p \frac{1}{2}\left(p^{2}+M^{2}+\frac{g^{2}}{96 \pi^{2}\left(\tilde{p}^{2}+1 / \Lambda^{2}\right)}-\frac{g^{2} M^{2}}{96 \pi^{2}} \ln \left(\frac{1}{M^{2}\left(\tilde{p}^{2}+1 / \Lambda^{2}\right)}\right)+\ldots\right) \phi(p) \phi(-p),
$$

where $M$ is the renormalized mass. Let us take the limits $\Lambda \rightarrow \infty$ and $\tilde{p} \rightarrow 0$. If we take first $\tilde{p} \rightarrow 0$ then $\tilde{p}^{2}<<\frac{1}{\Lambda^{2}}$ and $\Lambda_{e f f}=\Lambda$ showing that we recover the effective commutative theory

$$
\int d^{4} p \frac{1}{2}\left(p^{2}+M^{2}\right) \phi(p) \phi(-p) .
$$

If, however, we take $\Lambda \rightarrow \infty$ then $\tilde{p}^{2}>>\frac{1}{\Lambda^{2}}$ and $\Lambda_{e f f}^{2}=\frac{1}{\tilde{p}^{2}}$ and we get

$$
\int d^{4} p \frac{1}{2}\left(p^{2}+M^{2}+\frac{g^{2}}{96 \pi^{2} \tilde{p}^{2}}-\frac{g^{2} M^{2}}{96 \pi^{2}} \ln \left(\frac{1}{M^{2} \tilde{p}^{2}}\right)+\ldots\right) \phi(p) \phi(-p),
$$

which is singular when $\tilde{p} \rightarrow 0$. This shows that the limit $\Lambda \rightarrow \infty$ does not commute with the low momentum limit $\tilde{p} \rightarrow 0$ so that there is a mixing of $\mathrm{UV}$ and IR limits.

The theory is renormalizable at one loop order if we do not take $\tilde{p} \rightarrow 0$. What about higher loop orders? Suppose we have insertions of one loop mass corrections. Eventually we will have to integrate over small values of $\tilde{p}$ which diverges when $\Lambda \rightarrow \infty$. Then we find an IR divergence in a massive theory. This combination of UV and IR divergences makes the theory non-renormalizable.

There are also examples of non-renormalizable theories already at one loop order [5]. For a complex scalar field with interaction $\phi^{*} \star \phi^{*} \star \phi \star \phi$ it is found that the theory is one-loop non-renormalizable while $\phi^{*} \star \phi \star \phi^{*} \star \phi$ gives a one loop renormalizable model.

Then the question is whether it would be possible to find a theory which is renormalizable to all loop orders. Since the UV/IR mixing appears at the level of quadratic divergences a candidate theory would be a supersymmetric theory because it does not have such divergences $[9,10]$. As we shall see this indeed happens.

\section{Noncommutative Zumino model}

The noncommutative Wess-Zumino model in $3+1$ dimensions [6] has the action 


$$
\begin{aligned}
\mathcal{L}_{0}= & \frac{1}{2} \partial^{\mu} A \partial_{\mu} A+\frac{1}{2} \partial^{\mu} B \partial_{\mu} B+\frac{1}{2} \bar{\psi} i \not \partial \psi, \\
\mathcal{L}_{m}= & \frac{1}{2} F^{2}+\frac{1}{2} G^{2}+m F A+m G B-\frac{1}{2} m \bar{\psi} \psi, \\
\mathcal{L}_{g}= & g(F \star A \star A-F \star B \star B+G \star A \star B+G \star B \star A- \\
& \left.\bar{\psi} \star \psi \star A-\bar{\psi} \star i \gamma_{5} \psi \star B\right),
\end{aligned}
$$

where $A$ and $B$ are bosonic fields, $F$ and $G$ are auxiliary fields and $\psi$ is a Majorana spinor. The action is invariant under the usual supersymmetry transformations. They are not modified by the Moyal product since they are linear in the fields. The elimination of the auxiliary fields through their equations of motion produces quartic interactions. In terms of the complex field $\phi=A+i B$ we get $\phi^{*} \star \phi^{*} \star \phi \star \phi$ which is nonrenormalizable in the noncommutative case. This casts doubts about the renormalizability of the model but as we shall see supersymmetry saves the day.

As usual, the propagators are not modified by noncommutativity due to the property $\mathrm{d}$ ). They are given by

$$
\Delta_{A A}(p)=\Delta(p) \equiv \frac{i}{p^{2}-m^{2}+i \epsilon},
$$

$$
\begin{aligned}
\Delta_{F F}(p) & =p^{2} \Delta(p), \\
\Delta_{A F}(p) & =\Delta_{F A}(p)=-m \Delta(p), \\
S(p) & =\frac{i}{\not p-m} .
\end{aligned}
$$

Taking into account the symmetries the vertices are

$$
\begin{array}{lll}
F A^{2} & \text { vextex: } & i g \cos \left(p_{1} \wedge p_{2}\right), \\
F B^{2} & \text { vextex: } & -i g \cos \left(p_{1} \wedge p_{2}\right), \\
G A B & \text { vertex: } & 2 i g \cos \left(p_{1} \wedge p_{2}\right), \\
\bar{\psi} \psi A & \text { vertex: } & -i g \cos \left(p_{1} \wedge p_{2}\right), \\
\bar{\psi} \psi B & \text { vertex: } & -i g \gamma_{5} \cos \left(p_{1} \wedge p_{2}\right) .
\end{array}
$$

The degree of superficial divergence for a generic 1PI graph $\gamma$ is then

$$
d(\gamma)=4-I_{A F}-I_{B F}-N_{A}-N_{B}-2 N_{F}-2 N_{G}-\frac{3}{2} N_{\psi},
$$

where $N_{\mathcal{O}}$ denotes the number of external lines associated to the field $\mathcal{O}$ and $I_{A F}$ and $I_{B F}$ are the numbers of internal lines associated to the mixed propagators $A F$ and $B F$, respectively. In all cases we will regularize the divergent Feynman integrals by assuming that a supersymmetric regularization scheme does exist.

The one loop analysis can be done in a straightforward way. As in the commutative case all tadpoles contributions add up to zero. We have verified this explicitly. The self-energy of $A$ can be computed and the divergent part is contained in the integral

$$
16 g^{2} \int \frac{d^{4} k}{(2 \pi)^{4}}\left(1+\frac{1}{2} \cos (k \wedge p)\right) \frac{(p \cdot k)^{2}}{\left(k^{2}-m^{2}\right)^{3}} .
$$

The first term is logarithmically divergent. It differs by a factor 2 from the commutative case. As usual, this divergence is eliminated by a wave function renormalization. The second term is UV convergent and for small $p$ it behaves as $p^{2} \ln \left(p^{2} / m^{2}\right)$ and actually vanishes for $p=0$. Then there is no IR pole. The same analysis can be carried out for the others fields. For $F$ we find that the divergent part is

$$
4 g^{2} \int \frac{d^{4} k}{(2 \pi)^{4}}\left(1+\frac{1}{2} \cos (k \wedge p)\right) \frac{1}{\left(k^{2}-m^{2}\right)^{2}} \text {. }
$$

The first term is logarithmically divergent and can also be eliminated by a wave function renormalization. The second term diverges as $\ln \left(p^{2} / m^{2}\right)$ as $p$ goes to zero. However its multiple insertions is harmless. For the fermion field the divergent part is similar to the former results and needs also a wave function renormalization. 
The term containing $\cos (k \wedge p)$ behaves as $\not p \ln \left(p^{2} / m^{2}\right)$ and vanishes as $p$ goes to zero. Therefore, there is no UV/IR mixing in the self-energy as expected.

To show that the model is renormalizable we must also look into the interactions vertices. The $A^{3}$ vertex has no divergent parts as in the commutative case. The same happens for the other three point functions. For the four point vertices no divergence is found as in the commutative case. Hence, the noncommutative Wess-Zumino model is renormalizable at one loop with a wave-function renormalization and no UV/IR mixing.

To go to higher loop orders we proceed as in the commutative case [11]. We derived the supersymmetry Ward identities for the n-point vertex function. Then we showed that there is a renormalization prescription which is consistent with the Ward identities. They are the same as in the commutative case. And finally we fixed the primitively divergent vertex functions. Then we found that there is only a common wave function renormalization as in the commutative case. In general we expect

$$
\varphi_{R}=Z^{-1 / 2} \varphi, \quad m_{R}=Z m+\delta m, \quad g_{R}=Z^{3 / 2} Z^{\prime} g .
$$

At one loop we found $\delta m=0$ and $Z^{\prime}=1$. We showed that this also holds to all orders and no mass renormalization is needed.

Being the only consistent noncommutative quantum field theory in $3+1$ dimensions known so far it is natural to study it in more detail. As a first step in this direction we considered the nonrelativistic limit of the noncommutative Wess-Zumino model [12]. We found the low energy effective potential mediating the fermion-fermion and boson-boson elastic scattering in the nonrelativistic regime. Since noncommutativity breaks Lorentz invariance we formulated the theory in the center of mass frame of reference where the dynamics simplifies considerably. For the fermions we found that the potential is significatively changed by the noncommutativity while no modification was found for the bosonic sector. The modifications found give rise to an anisotropic differential cross section.

\section{Noncommutative Gross- Neveu and Nonlinear Sigma Models}

Another model where nonrenormalizability is spoiled by the noncommutativity is the $O(N)$ Gross-Neveu model. This model is perturbatively renormalizable in $1+1$ dimensions and $1 / N$ renormalizable in $1+1$ and $2+1$ dimensions. In both cases it presents dynamical mass generation. It is described by the Lagrangian

$$
\mathcal{L}=\frac{i}{2} \bar{\psi}_{i} \not \partial \psi_{i}+\frac{g}{4 N}\left(\bar{\psi}_{i} \psi_{i}\right)\left(\bar{\psi}_{j} \psi_{j}\right)
$$

where $\psi_{i}, i=1, \ldots N$, are two-component Majorana spinors. Since it is renormalizable in the $1 / N$ expansion in $1+1$ and $2+1$ dimensions we will consider both cases. As usual, we introduce an auxiliary field $\sigma$ and the Lagrangian turns into

$$
\mathcal{L}=\frac{i}{2} \bar{\psi}_{i} \not \partial \psi_{i}-\frac{\sigma}{2}\left(\bar{\psi}_{i} \psi_{i}\right)-\frac{N}{4 g} \sigma^{2}
$$

Replacing $\sigma$ by $\sigma+M$ where $M$ is the VEV of the original $\sigma$ we get the gap equation (in Euclidean space)

$$
\frac{M}{2 g}-\int \frac{d^{D} k}{(2 \pi)^{D}} \frac{M}{k_{E}^{2}+M^{2}}=0 .
$$

To eliminate the UV divergence we need to renormalize the coupling constant by

$$
\frac{1}{g}=\frac{1}{g_{R}}+2 \int \frac{d^{D} k}{(2 \pi)^{D}} \frac{1}{k_{E}^{2}+\mu^{2}} .
$$

In $2+1$ dimensions we find

$$
\frac{1}{g_{R}}=\frac{\mu-|M|}{2 \pi}
$$

and therefore only for $-\frac{1}{g_{R}}+\frac{\mu}{2 \pi}>0$ it is possible to have $M \neq 0$, otherwise $M$ is necessarily zero. No such a restriction exists in $1+1$ dimensions. In any case, we will focus only in the massive phase. The propagator for $\sigma$ is proportional to the inverse of the following expression

$$
-\frac{i N}{2 g}-i N \int \frac{d^{D} k}{(2 \pi)^{D}} \frac{k \cdot(k+p)+M^{2}}{\left(k^{2}-M^{2}\right)\left[(k+p)^{2}-M^{2}\right]},
$$

which is divergent. Taking into account the gap equation the above expression reduces to

$$
\frac{\left(p^{2}-4 M^{2}\right) N}{2} \int \frac{d^{D} k}{(2 \pi)^{D}} \frac{1}{\left(k^{2}-M^{2}\right)\left[(k+p)^{2}-M^{2}\right]},
$$

which is finite. Then there is a fine tuning which is responsible for the elimination of the divergence and which might be absent in the noncommutative case due to the UV/IR mixing. 
The noncommutative model is defined by

$$
S_{G N}=\int d^{D} x\left[\frac{i}{2} \bar{\psi} \not \partial \psi-\frac{M}{2} \bar{\psi} \psi-\frac{1}{2} \sigma \star(\bar{\psi} \star \psi)-\frac{N}{4 g} \sigma^{2}-\frac{N}{2 g} M \sigma\right] .
$$

Elimination of the auxiliary field results in a fourfermion interaction of the type $\bar{\psi}_{i} \star \psi_{i} \star \bar{\psi}_{j} \star \psi_{j}$. However a more general four-fermion interaction may involve a term like $\bar{\psi}_{i} \star \bar{\psi}_{j} \star \psi_{i} \star \psi_{j}$. This last combination does not have a simple $1 / N$ expansion and we will not con- sider it. The Moyal product does not affect the propagators and the trilinear vertex acquires a correction of $\cos \left(p_{1} \wedge p_{2}\right)$ with regard to the commutative case. Hence the gap equation is not modified, while the propagator for the $\sigma$ is now proportional to the inverse of

$$
-\frac{i N}{2 g}-N \int \frac{d^{D} k}{(2 \pi)^{D}} \cos ^{2}(k \wedge p) \frac{k \cdot(k+p)+M^{2}}{\left(k^{2}-M^{2}\right)\left[(k+p)^{2}-M^{2}\right]} .
$$

Now the divergent part is no longer canceled and this turns the model into a nonrenormalizable one.

On the other side, the nonlinear sigma model also presents troubles in its noncommutative version. The noncommutative model is described by

$$
\mathcal{L}=-\frac{1}{2} \varphi_{i}\left(\partial^{2}+M^{2}\right) \varphi_{i}+\frac{1}{2} \lambda \star \varphi_{i} \star \varphi_{i}-\frac{N}{2 g} \lambda,
$$

where $\varphi_{i}, i=1, \ldots, N$, are real scalar fields, $\lambda$ is the auxiliary field and $M$ is the generated mass. The leading correction to the $\varphi$ self-energy is

$$
-i \int \frac{d^{2} k}{(2 \pi)^{2}} \frac{\cos ^{2}(k \wedge p)}{(k+p)^{2}-M^{2}} \Delta_{\lambda}(k),
$$

where $\Delta_{\lambda}$ is the propagator for $\lambda$. As for the case of the scalar field this can be decomposed as a sum of a quadratically divergent part and a UV finite part. Again there is the $\mathrm{UV} / \mathrm{IR}$ mixing destroying the $1 / N$ expansion.

\section{Noncommutative Super- symmetric Nonlinear Sigma Model}

The Lagrangian for the commutative supersymmetric sigma model is given by

$$
\mathcal{L}=\frac{1}{2} \partial^{\mu} \varphi_{i} \partial_{\mu} \varphi_{i}+\frac{i}{2} \bar{\psi}_{i} \not \partial \psi_{i}+\frac{1}{2} F_{i} F_{i}+\sigma \varphi_{i} F_{i}+\frac{1}{2} \lambda \varphi_{i} \varphi_{i}-\frac{1}{2} \sigma \bar{\psi}_{i} \psi_{i}-\bar{\xi} \psi_{i} \varphi_{i}-\frac{N}{2 g} \lambda
$$

where $F_{i}, i=1, \ldots, N$, are auxiliary fields. Furthermore, $\sigma, \lambda$ and $\xi$ are the Lagrange multipliers which implement the supersymmetric constraints. After the change of variables $\lambda \rightarrow \lambda+2 M \sigma, F \rightarrow F-M \varphi$ where $M=<\sigma>$, and the shifts $\sigma \rightarrow \sigma+M$ and $\lambda \rightarrow \lambda+\lambda_{0}$, where $\lambda_{0}=\langle\lambda\rangle$, we arrive at a more symmetric form for the Lagrangian

$$
\begin{aligned}
\mathcal{L}= & -\frac{1}{2} \varphi_{i}\left(\partial^{2}+M^{2}\right) \varphi_{i}+\frac{1}{2} \bar{\psi}_{i}(i \not \partial-M) \psi_{i}+\frac{1}{2} F_{i}^{2}+M^{2} \varphi_{i}^{2}+\frac{1}{2} \lambda_{0} \varphi_{i}^{2} \\
& +\frac{1}{2} \lambda \varphi_{i}^{2}+\sigma \varphi_{i} F_{i}-\frac{1}{2} \sigma \bar{\psi}_{i} \psi_{i}-\bar{\xi} \psi_{i} \varphi_{i}-\frac{N}{2 g} \lambda-\frac{N}{g} M \sigma .
\end{aligned}
$$

Now supersymmetry requires $\lambda_{0}=-2 M^{2}$ and the gap equation is

$$
\int \frac{d^{D} k}{(2 \pi)^{D}} \frac{i}{k^{2}-M^{2}}=\frac{1}{g}
$$


so a coupling constant renormalization is required. We now must examine whether the propagator for $\sigma$ depends on the this renormalization. We find that the two point function for $\sigma$ is proportional to the inverse of

$$
\frac{\left(p^{2}-4 M^{2}\right) N}{2} \int \frac{d^{D} k}{(2 \pi)^{D}} \frac{1}{\left(k^{2}-M^{2}\right)\left[(k+p)^{2}-M^{2}\right]},
$$

which is identical to the Gross-Neveu case. Notice that the gap equation was not used. The finiteness of the above expression is a consequence of supersymmetry.

The noncommutative version of the supersymmetric nonlinear sigma model is given by

$$
\begin{aligned}
\mathcal{L}= & -\frac{1}{2} \varphi_{i}\left(\partial^{2}+M^{2}\right) \varphi_{i}+\frac{1}{2} \bar{\psi}_{i}(i \not \partial-M) \psi_{i}+\frac{1}{2} F_{i}^{2}+\frac{\lambda}{2} \star \varphi_{i} \star \varphi_{i} \\
& -\frac{1}{2} F_{i} \star\left(\sigma \star \varphi_{i}+\varphi_{i} \star \sigma\right)-\frac{1}{2} \sigma \star \bar{\psi}_{i} \star \psi_{i}-\frac{1}{2}\left(\bar{\xi} \star \psi_{i} \star \varphi_{i}+\bar{\xi} \star \varphi_{i} \star \psi_{i}\right) \\
& -\frac{N}{2 g} \lambda-\frac{N M \sigma}{g} .
\end{aligned}
$$

Notice that supersymmetry dictates the form of the trilinear vertices. Also, the supersymmetry transformations are not modified by noncommutativity since they are linear and no Moyal products are required.

The propagators are the same as in the commutative case. The vertices have cosine factors due to the Moyal product

$$
\begin{array}{ccl}
\lambda \varphi^{2} & \text { vertex: } & \frac{i}{2} \cos \left(p_{1} \wedge p_{2}\right), \\
\sigma \varphi F & \text { vertex: } & -i \cos \left(p_{1} \wedge p_{2}\right), \\
\bar{\psi} \psi \sigma & \text { vertex: } & -\frac{i}{2} \cos \left(p_{1} \wedge p_{2}\right), \\
\bar{\xi} \psi \varphi & \text { vertex: } & -i \cos \left(p_{1} \wedge p_{2}\right) .
\end{array}
$$

We again consider the propagators for the Lagrange multiplier fields. Now the $\sigma$ propagator is modified by the cosine factors and is proportional to the inverse of

$$
\frac{\left(p^{2}-4 M^{2}\right) N}{2} \int \frac{d^{D} k}{(2 \pi)^{D}} \frac{\cos ^{2}(k \wedge p)}{\left(k^{2}-M^{2}\right)\left[(k+p)^{2}-M^{2}\right]} .
$$

It is well behaved both in UV and IR regions. The propagators for $\lambda$ and $\xi$ are proportional to the inverse of

$$
\frac{N}{2} \int \frac{d^{D} k}{(2 \pi)^{D}} \cos ^{2}(k \wedge p) \frac{1}{\left[(k+p)^{2}-M^{2}\right]\left[k^{2}-M^{2}\right]},
$$

and

$$
N \frac{(\not p+2 M)}{2} \int \frac{d^{D} k}{(2 \pi)^{D}} \cos ^{2}(k \wedge p) \frac{1}{\left[(k+p)^{2}-M^{2}\right]\left[k^{2}-M^{2}\right]},
$$

respectively. They are also well behaved in UV and IR regions.

The degree of superficial divergence for a generic 1PI graph $\gamma$ is

$$
d(\gamma)=D-\frac{(D-1)}{2} N_{\psi}-\frac{(D-2)}{2} N_{\varphi}-\frac{D}{2} N_{F}-N_{\sigma}-\frac{3}{2} N_{\xi}-2 N_{\lambda}
$$

where $N_{\mathcal{O}}$ is the number of external lines associated to the field $\mathcal{O}$. Potentially dangerous diagrams are those contributing to the self-energies of the $\varphi$ and $\psi$ fields since, in principle, they are quadratic and linearly divergent, respectively. For the self-energies of $\varphi$ and $\psi$ we find that they diverge logarithmically and they can be removed by a wave function renormalization of the respective field. The same happens for the auxiliary field $F$. The renormalization factors for them are the same so supersymmetry is preserved in the noncommutative theory. This analysis can be extended to the n-point functions. In $2+1$ dimensions we find nothing new showing the renormalizability of the model at 
leading order of $1 / N$. However, in $1+1$ dimensions there some peculiarities. Since the scalar field is dimensionless in $1+1$ dimensions any graph involving an arbitrary number of external $\varphi$ lines is quadratically divergent. In the four-point function there is a partial cancellation of divergences but a logarithmic divergence still survives. The counterterm needed to remove it can not be written in terms of $\int d^{2} x \varphi_{i} \star \varphi_{i} \star \varphi_{j} \star \varphi_{j}$ and $\int d^{2} x \varphi_{i} \star \varphi_{j} \star \varphi_{i} \star \varphi_{j}$. A possible way to remove this divergence is by generalizing the definition of $1 \mathrm{PI}$ diagram along the lines suggested in [13] for the commutative nonlinear sigma model. However the cosine factors do not allow us to use this mechanism which casts doubt about the renormalizability of the noncommutative supersymmetric $O(N)$ nonlinear sigma model in $1+1$ dimensions.

\section{Conclusions}

We have shown that it is possible to build consistent quantum field theories in noncommutative space. It seems that supersymmetry is an essential ingredient for renormalizability. The models studied here do not involve gauge fields and this considerably simplifies the situation. All vertices are deformed in the same way by the Moyal product and this was essential to analyze the amplitudes. With gauge fields the situation is much more complicated because the vertices are deformed in different ways. However, supersymmetric gauge theories may still have a better behavior.

\section{Acknowledgments}

This work was done in collaboration with $\mathrm{H}$. O. Girotti, M. Gomes and A. J. da Silva. It was partially supported by Fundação de Amparo à Pesquisa do Estado de São Paulo (FAPESP), Conselho Nacional de Desenvolvimento Científico e Tecnológico (CNPq), and PRONEX under contract CNPq 66.2002/1998-99.

\section{References}

[1] N. Seiberg and E. Witten, "String Theory and Noncommutative Geometry", hep-th/9908142, JHEP 9909, 032 (1999).

[2] T. Filk, Phys.Lett. B 376, 53 (1996).

[3] S. Minwalla, M. Van Raamsdonk and N. Seiberg, "Noncommutative Perturbative Dynamics", hepth/9912072.

[4] I. Y. Aref'eva, D. M. Belov and A. S. Koshelev, "Twoloop Diagrams in Noncommutative $\phi_{4}^{4}$ Theory", hepth/9912075.

[5] I. Y. Aref'eva, D. M. Belov and A. S. Koshelev, "A note on UV/IR for Noncommutative Complex Scalar Field", hep-th/0001215.

[6] H. O. Girotti, M. Gomes, V. O. Rivelles, A. J. da Silva, "A Consistent Noncommutative Field Theory: the Wess-Zumino Model", hep-th/0005272, Nucl.Phys. B 587, 299 (2000).

[7] H. O. Girotti, M. Gomes, V. O. Rivelles, A. J. da Silva, "The Noncommutative Supersymmetric Nonlinear Sigma Model", hep-th/0102101.

[8] N. Seiberg, L. Susskind, N. Toumbas, "Space/Time Non-Commutativity and Causality", hep-th/0005015, JHEP 0006 (2000) 044.

[9] I. Chepelev and R. Roiban, "Renormalization of Quantum field Theories on Noncommutative $R^{d}$. I: Scalars," hep-th/9911098.

[10] S. Ferrara and M. A. Lledo, "Some aspects of Deformations of Supersymmetric Field Theories," hepth/0002084.

[11] J. Iliopoulos and B. Zumino, "Broken supergauge symmetry and renormalization", Nucl. Phys. B 76, 310 (1974).

[12] H.O. Girotti, M. Gomes, V.O. Rivelles, A.J. da Silva, "The Low Energy Limit of the Noncommutative WessZumino Model", hep-th/0101159.

[13] I. Ya. Aref'eva, Theor. Math. Phys. 36, 573 (1979); Ann. Phys. (NY) 117, 393 (1979); I. Ya. Aref'eva, E. R. Nissimov and S. J. Pacheva, Commun. Math. Phys. 71, 213 (1980). See also J. H. Lowenstein and E. R. Speer, Nucl.. Phys. B 158, 397 (1979). 